\title{
Deep electron trap level in semi-insulating GaAs
}

\author{
A K SAXENA \\ Department of Electronics and Communication Engineering, University of Roorkee, Roorkee \\ 247667 , India \\ MS received 19 October 1984

\begin{abstract}
The experimental data on the Hall measurements have been used to characterize deep electron trapping levels in $\mathrm{Cr}$ doped semi-insulating $\mathrm{GaAs}$ crystals. The energy of the level below the conduction band edge has been found to be $\sim 0.8 \mathrm{eV}$ and is thought to be related to $\mathrm{Ga}$ vacancies in the host crystal and $\mathrm{Cr}$ impurities.
\end{abstract}

Keywords. Semiconductor devices and materials; semiconductors (III-V); Hall measurements; electron traps.

Transport properties of $\mathrm{GaAs}$ and $\mathrm{Ga}_{1-x} \mathrm{Al}_{x}$ As alloys are important because of their potential applications in a variety of semiconductor devices both optical and microwave. The author has previously reported the interpretation and analysis of the Hall electron concentration $n_{H}$ (Saxena 1982) and the capacitance measurements on the Schottky Barrier diodes (Bhattacharya et al 1979) to characterize various energy levels in the band gap of $\mathrm{Ga}_{1-x} \mathrm{Al}_{x}$ As crystals, supported by semi-insulating (sI) GaAs substrates. From the point of view of device applications such as FET and integrated circuits, identification and characterization of energy levels in si GaAs is extremely important. Although VPE, LPE and bulk GaAs crystals have been studied (Hasegawa and Majerfeld 1975), not much is known about the traps in sI GaAs. In the present letter, we present results on the temperature dependence of $n_{H}$ for $\mathrm{Ga}_{1-x} \mathrm{Al}_{x} \mathrm{As} / \mathrm{GaAs}$ crystals to identify traps in si GaAs.

The high purity $\mathrm{Ga}_{1-x} \mathrm{Al}_{x}$ As layers were grown on $\mathrm{Cr}$ doped sl GaAs substrates by LPE. The Hall measurements were made on standard Clover leaf samples and in a magnetic field of 5 kgauss. Sn was used to make the ohmic contacts by firing them in an $\mathrm{H}_{2}$ atmosphere for $\sim 3$ minutes at $500^{\circ} \mathrm{C}$. The temperature of the sample could be stabilized to within $\pm 1 \mathrm{~K}$ and a high impedance electrometer was used to measure the signals through the samples. To account for the finite size of the contacts, correction factors as given by Van-Der Pauw (1958/59) were applied to calculate $n_{H}$ from the measured values.

The variation of $n_{H}$ with temperature for a typical $\mathrm{Ga}_{1-x} \mathrm{Al}_{x} \mathrm{As}(X=0.23)$ layer, supported by SI GaAs substrate is shown in figure 1 by full squares. The decrease in $n_{H}$ below $300 \mathrm{~K}$ is due to electron 'freeze out' to a non-shallow level in the alloy while in the temperature interval $300 \leqslant T \leqslant 500 \mathrm{~K}$, it is due to intervalley electron transfer from the central to the satellite minima. For $T \gtrsim 500 \mathrm{~K}, n_{H}$ increased sharply with temperature. This could be either due to (i) ionization of deep energy levels in the band gap of the alloy, or (ii) due to the electron conduction in the sı GaAs substrate. Next the epitaxial layers were removed by chemical etching and measurements repeated on si GaAs only. 


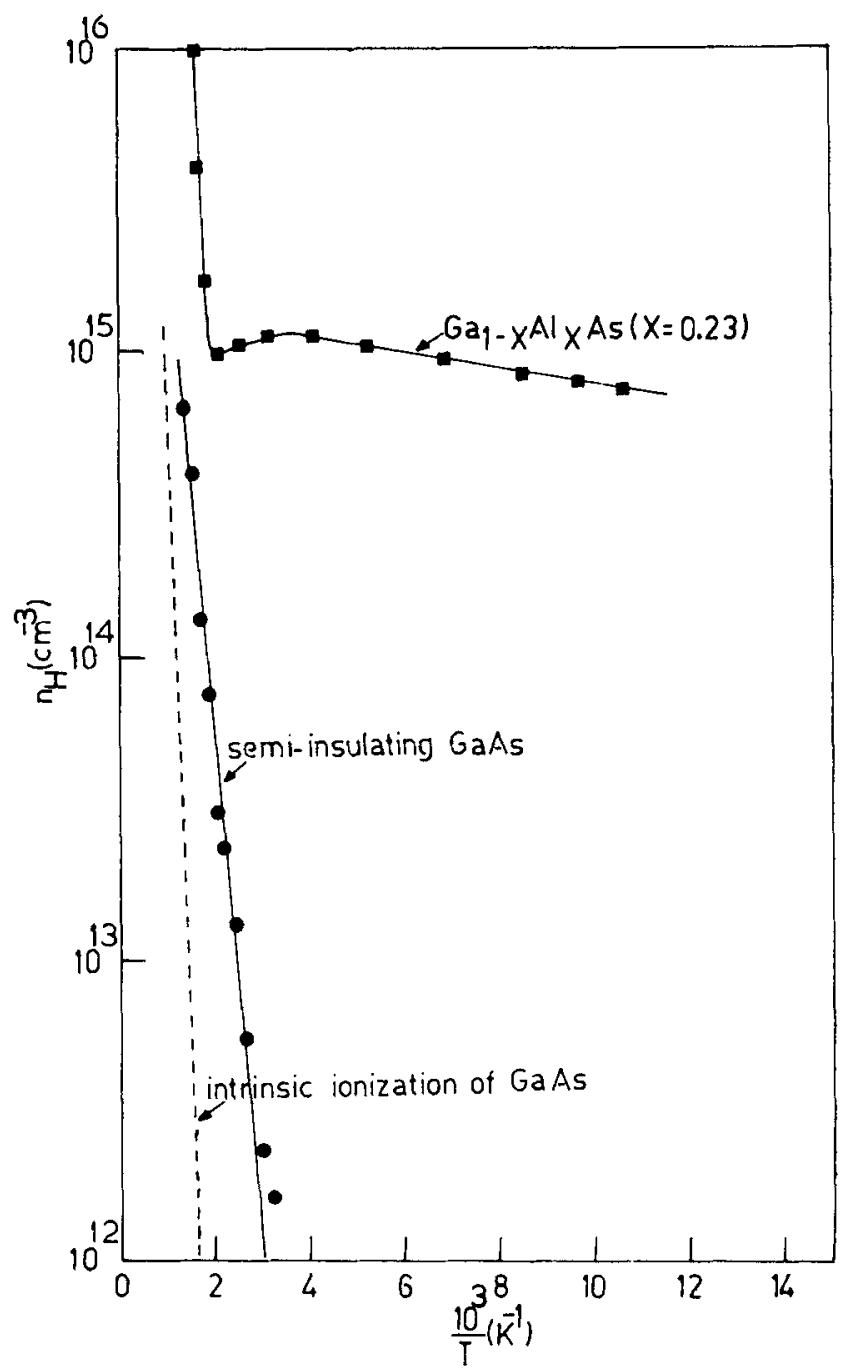

Figure 1. Temperature dependence of the Hall electron concentration $n_{H}$ for LPE $\mathrm{Ga}_{1-x} \mathrm{Al}_{x}$ As alloy $(x=0-23)(\boldsymbol{C})$ and semi-insulating $\mathrm{GaAs}$ substrate (c). Also shown (broken line) is the calculated $n_{H}$ due to the intrinsic ionization of GaAs.

The results so obtained are also shown in figure 1 by full circles.

The Hall concentration is calculated from the equation (Van-Der Pauw 1958/59)

$$
n_{H}=B /(q t \Delta R) \text {, }
$$

where $B=$ magnetic field strength, $t=$ thickness of the layer on which measurements are made, $q=$ electronic charge and $\Delta R=$ change in transfer resistance of the layer on the application of the magnetic field. From (1), it follows that

$$
\frac{\left(n_{H}\right)_{e}}{\left(n_{H}\right)_{s}}=\frac{t_{s} \Delta R_{s}}{t_{e} \Delta R_{e}}
$$


where the subscripts $e$ and $s$ represent the epitaxial layer and substrate, respectively. Since the parameters $t$ and $\Delta R$ are known experimentally, we find that in the temperature interval $500 \leqslant T \leqslant 750 \mathrm{~K}$, the ratio given by 2 is 35 . On the other hand, the measured value of this ratio as obtained from figure 1 is also $\sim 35$ for the same temperature interval. Thus, the abrupt rise in $n_{H}$ for $T \gtrsim 500 \mathrm{~K}$ is due to the electron conduction in the sI GaAs.

In order to analyse the data for $\mathrm{Ga}_{1-x} \mathrm{Al}_{\boldsymbol{x}} \mathrm{As}$ layers and to account for the abrupt rise in $n_{H}$ at high temperatures, an electron level with activation energy of $\sim 0.8 \mathrm{eV}$ was needed (Saxena 1982). However, using capacitance measurements on Schottky Barrier diodes, we could not detect any level in $\mathrm{Ga}_{1-x} \mathrm{Al}_{x}$ As layers with such a large activation energy (Bhattacharya et al 1979). This further supports our conclusion that the rise in $n_{H}$ at high temperatures is indeed due to the electron conduction in SI GaAs and not due to such deep energy levels in $\mathrm{Ga}_{1-x} \mathrm{Al}_{x}$ As layers.

The next question arises about the possible conduction mechanism in SI GaAs at high temperatures. There could be two possibilities again namely (1) ionization of a deep energy level and (2) intrinsic ionization of GaAs.

We have calculated $n_{H}$ due to intrinsic-ionization from the following equations (Saxena 1982)

$$
n_{H}=\left[n_{1}\left(1+\frac{n_{2}}{n_{1}} \frac{\mu_{2}}{\mu_{1}}\right)^{2}\right] /\left[\left(1+\frac{n_{2}}{n_{1}} \frac{\mu_{2}^{2}}{\mu_{1}^{2}}\right)\right]
$$

where $\quad \frac{n_{2}}{n_{1}}=\left(\frac{N_{C 2}}{N_{C 1}}\right)^{3 / 2} \exp \left[-\Delta E_{12} / K T\right]$

$$
n=n_{1}+n_{2}=N_{C 1} \exp \left[-E_{G} / 2 K T\right]
$$

and

$$
N_{\mathrm{C}}=\left(4 \sqrt{2} / h^{3}\right)\left(\pi m_{1,2} K T\right)^{3 / 2}
$$

Here the subscripts 1 and 2 refer to the central and satellite minima, respectively. $\Delta E$ is the sub-band gap between these minima and $E_{G}$, the band gap. Considering $m_{1}$ $=0.067 m_{0}, m_{2}=0.55 m_{0}, E_{G}=1.425 \mathrm{eV}, \Delta E_{12}=0.285 \mathrm{eV}$ and $\mu_{1} / \mu_{2} \simeq 8$ (Saxena 1982), $n_{H}$ is calculated and the results are shown in figure 1 by the broken line. It is evident that the slope of the $n_{H}$ curve for intrinsic ionization is different than for the measured $n_{H}$ for GaAs. Also the intrinsic ionization occurs at much higher temperatures than the observed $n_{H}$ for GaAs. Hence, the abrupt rise in $n_{H}$ at high temperatures could not be due to intrinsic ionization. A computer analysis of the Hall data for $\mathrm{Ga}_{1-x} \mathrm{Al} \mathrm{l}_{x} \mathrm{As}$ layers indeed requires a deep level with an energy of $\sim 0.8 \mathrm{eV}$, which now turns out to be the activation energy of a level in si GaAs.

It is interesting to note that an electron trap level with an activation energy of $0.83 \mathrm{eV}$ is present in $n$-type VPE and bulk GaAs (Hasegawa and Majerfeld 1975) and also in Cr-doped $n$-type LPE GaAs (Martin et al 1977). Compared to shallow donor density, the trap density has been found to be much larger in bulk GaAs, as expected, since the $0.83 \mathrm{eV}$ trap level has been shown to be related to $\mathrm{Ga}$ vacancies in the host crystal (Hasegawa and Majerfeld 1976). Thus, it is tempting to think that the level observed in si GaAs in the present work is also related to a complex of $\mathrm{Ga}$ vacancies and $\mathrm{Cr}$ impurities. We would have liked to further verify our results by measuring $n_{H}$ on pure epitaxial layers of $\mathrm{Ga}_{1-x} \mathrm{Al}_{x}$ As without substrates but this could not be done due to the very small thickness $(\sim 5 \mu \mathrm{m})$ of these layers. 


\section{Acknowledgements}

I wish to thank the Royal Society of London for offering a visiting Fellowship and to Dr I G A Davies of Standard Telecommunication Laboratory for providing the crystals. The financial assistance from the DST, INSA, Kothari Scientific and Research Institute and University of Roorkee are gratefully acknowledged.

\section{References}

Bhattacharya P K, Majerfeld A and Saxena A K 1979 Proceedings of the 6th International Symposium on gallium arsenide and related compounds, St. Louis (London: Institute of Physics) pp. 199-210.

Hasegawa F and Majerfeld A 1976 Electron. Lett. 1252

Hasegawa $F$ and Majerfeld A 1975 Electron. Lett. 11286

Martin G M, Mitonneau A and Mircea A 1977 Electron. Lett. 13191

Saxena A K 1982 Phys. Status Solidi B77 777

Van-Der Pauw L J 1958/59 Philips Tech. Rev. 20220 